\title{
Calidad y acreditación de los servicios de urgencias
}

\section{Quality and accreditation of emergency departments}

\author{
T. Belzunegui' ${ }^{1}$, P. Busca ${ }^{2}$, L. López-Andújar ${ }^{3}$, M. Tejedor ${ }^{4}$
}

\section{RESUMEN}

El concepto de calidad está ligado al mundo sanitario desde muy antiguo y es uno de los elementos estratégicos en que se fundamenta la transformación y mejora de los sistemas sanitarios modernos. El estudio de la calidad en la asistencia sanitaria supone abordajes diversos dado que entraña significados distintos para los pacientes, profesionales y gestores. En los últimos años se están introduciendo, de forma progresiva, sistemas formales de gestión de calidad, como son la certificación según las Normas ISO-9000, el sistema de acreditación de la Joint Commission on Accreditation of Healthcare Organizations (JCAHO) y el modelo europeo de excelencia (EFQM).

La gestión por procesos es otra herramienta encaminada a conseguir los objetivos de calidad total en el proceso asistencial y abordar los problemas de salud desde una visión centrada en el paciente. Una asistencia sanitaria de calidad es aquella que se proporciona con una práctica clínica basada en pruebas, conforme a los conocimientos científicos actuales, siguiendo guías clínicas elaboradas con criterios de medicina basada en la evidencia, con el menor riesgo para los pacientes y los familiares, con la mayor eficiencia y con la mayor satisfacción para usuarios y el personal sanitario.

La acreditación de centros y servicios sanitarios es, sin duda, el método de garantía de calidad más aceptado. Podemos definirlo como el proceso de evaluación externa al que se somete una organización sanitaria, basado en la revisión de unos criterios o estándares consensuados, cuyo cumplimiento manifiesta que ese centro desarrolla su funciones con parámetros de calidad, al menos, cercanos a la excelencia.

Palabras clave. Calidad asistencial. Urgencias. Acreditación. Gestión clínica. Indicadores.

\begin{abstract}
The concept of quality has been linked to the health care world since the remote past and is one of the strategic elements on which the transformation and improvement of modern health systems is based. Study of quality in health care involves different approaches given that it holds different meanings for patients, professionals and managers. In recent years formal systems of quality management have been progressively introduced, such as certification according to the ISO-9000 Norms, the system of accreditation of the Joint Commission on Accreditation of Healthcare Organizations (JCAHO) and the European Model of Excellence (EFQM).
\end{abstract}

Management by processes is another tool directed towards achieving the aims of total quality in the care process and tackling health problems centred on the patient. Quality health care involves evidence based clinical practice; based on current scientific knowledge, following clinical practice guidelines based on evidence, with the least risk for patients and relatives, involving greater efficiency and greater satisfaction for users and health personnel.

The accreditation of health care centres and services is, without doubt, the most widely accepted method for guaranteeing quality. It can be defined as the process of external evaluation to which a health organisation is subjected, based on a review of certain accepted criteria and standards, whose fulfilment demonstrates that that centre is developing its functions within parameters of quality that at least approach excellence.

Key words. Health care. Emergency care. Accreditation. Clinical management. Indicators.

\section{Servicio de Urgencias. Hospital de Navarra.} Pamplona.

2. Servicio de Urgencias. Hospital Donostia. San Sebastián.

3. Hospital General Universitario. Alicante.

4. Hospital Universitario «Reina Sofía». Córdoba.

\author{
Correspondencia \\ Tomás Belzunegui Otano \\ Médico Adjunto Servicio de Urgencias \\ Hospital de Navarra \\ Irunlarrea, 3 \\ 31008 Pamplona \\ E-mail: tomas.belzunegui@unavarra.es
}




\section{INTRODUCCIÓN}

El día 14 de enero de 2009 la revista New England Journal of Medicine publicó un artículo en el que, tras un largo estudio llevado a cabo en 8 hospitales de 8 países diferentes, se demostró que la utilización de un listado de verificación de la seguridad quirúrgica reducía la mortalidad y la morbilidad (complicaciones) ligada a la cirugía de una forma altamente significativa, aproximadamente un $30 \%{ }^{1}$. ¿Qué importancia y significado tiene esto? La respuesta es que a partir de determinados logros en los resultados clínicos, éstos no pueden mejorarse sólo con un incremento de las denominadas "competencias clínicas" tradicionales: el conocimiento de la etiopatogenia, la sintomatología, los medios diagnósticos y terapéuticos de una determinada entidad nosológica,... Todo esto no es suficiente para alcanzar unos resultados clínicos óptimos sino que deben articularse medidas e instrumentos para que aquellas competencias clínicas se apliquen sistemática y adecuadamente en la práctica y estas herramientas necesarias están relacionadas con mejoras de la planificación, organización y gestión de los procesos clínicos. En el estudio comentado se aseguró, mediante una lista de comprobación que obligó a ello, que se hacía siempre lo que se tiene que hacer: comprobar la identidad del paciente y el lado quirúrgico, el funcionamiento de los aparatos de anestesia y pulsioxímetro, que se le ha puesto el antibiótico profiláctico, que se cuentan las gasas y el material quirúrgico, que se etiquetan correctamente y se envían en tiempo las muestras a anatomía patológica, etc. Todas éstas son precisamente causas/fallos de los que hay evidencia que están en la raíz de la aparición de graves complicaciones derivadas de la cirugía.

El Estudio Nacional sobre los Efectos Adversos ligados a la Hospitalización (ENEAS) ha puesto de manifiesto que en España la incidencia de pacientes con efectos adversos (EA) relacionados con la asistencia sanitaria fue de $9,3 \%$. La incidencia de pacientes con EA relacionados directamente con la asistencia hospitalaria (excluidos los de atención primaria, consultas externas y los ocasionados en otro hospital) fue de $8,4 \%^{2}$.

La seguridad de los pacientes es, probablemente, el componente clave de la calidad de la asistencia ${ }^{3}$, pero no el único. Como clásicamente pusieron de manifiesto Donabedian, Vuhori y otros grandes maestros, la calidad asistencial tiene multitud de dimensiones: la rapidez en la atención, el nivel científico-técnico, la amabilidad, la satisfacción del paciente y otros.

La atención en los servicios de urgencias es un tema de importancia capital para millones de ciudadanos que acuden a ellas cada año en España. Los profesionales sanitarios asistimos con perplejidad a la resistencia que tienen las distintas administraciones sanitarias para colocar este tipo de asistencia entre las prioridades del sistema sanitario a pesar de la evidencia de lo evidente. Hasta tal punto es así que un hito histórico en la mejora de los servicios tuvo que ser desencadenado por un informe del Defensor del Pueblo Español y motivado por las continuas denuncias de los ciudadanos recibidas en aquella institución.

La magnitud del impacto de una mejora en la asistencia de urgencias es extraordinaria, tanto para la totalidad de los servicios de salud como socialmente. Esto debería animar a las autoridades sanitarias a llevar la iniciativa en este tema. Pero, ¿cómo abordar un asunto de tanto interés y complejidad? Probablemente esta pregunta no tiene una única respuesta, pero sin duda es una cuestión pertinente que debe plantearse el colectivo de profesionales comprometidos con esta modalidad de asistencia.

Desde un enfoque general o "macro" son claves los sistemas de acreditación de servicios ya que constituyen una herramienta útil para alcanzar una cierta homogeneidad en las estructuras, recursos disponibles, la planificación, la organización y gestión de los procesos asistenciales. Los estándares a aplicar precisan del consenso profesional, de su actualización y renovación permanentes y de la colaboración con las autoridades sanitarias españolas y de las distintas comunidades autónomas. 
¿Debe existir un sistema de clasificación de pacientes en todos los servicios de urgencias de hospitales? ¿Qué cualificación profesional es la idónea para aquellos profesionales médicos, enfermeros y técnicos que asisten a emergencias? ¿Cuál debe ser la capacitación de los profesionales en un servicio de emergencias? ¿Qué procesos clínicos deben estar protocolizados? ¿Qué requisitos mínimos estructurales y de dotación de equipamiento de electromedicina son convenientes que existan en un punto de atención continuada o en un dispositivo de cuidados críticos y urgencias?

Para responder a estas cuestiones, y por muy paradójico que pueda parecer, aún hoy no existe un consenso internacional ni nacional por las distintas administraciones sanitarias ni siquiera en la opinión técnica de los profesionales y de las organizaciones que los representan, no sólo en lo concerniente a la planificación y organización de los servicios sino en cómo desarrollar un sistema de calidad, aunque haya algunas experiencias publicadas aisladas ${ }^{4}$.

Por fortuna, varios grupos de profesionales españoles en el seno de la Sociedad Española de Medicina de Urgencias y Emergencias (SEMES) entendieron hace ya unos años la importancia de este asunto $\mathrm{y}$ han venido trabajando en ello. Esto ha permitido una serie de logros entre los que se encuentra un Sistema Español de Acreditación de Servicios de Urgencias de Hospitales, Emergencias y de Urgencias en Atención Primaria, que se comentan más adelante en este trabajo. Este sistema de acreditación ${ }^{5-9}$ nos está permitiendo, junto con otros grupos españoles ${ }^{10-12}$, aparecer en el contexto internacional con una voz y un lenguaje propios.

En estratos intermedios, en el nivel "meso" de las organizaciones, se necesitan instrumentos como el desarrollo de programas de gestión clínica o de calidad ${ }^{13-16}$ que, aunque se basan en la participación de todo el equipo, la responsabilidad máxima recae en los líderes formales, o sea, en los jefes de servicio, directores o coordinadores.

En la práctica, durante la asistencia cotidiana a los pacientes, en el nivel «micro", hay que disponer de herramientas sencillas que permitan medir el resultado de las actuaciones que posibilitan la autoevaluación y la mejora continuada de la atención que se presta. Los indicadores ${ }^{17}$ son aquí, por tanto, de una gran relevancia.

Por tanto, se precisa de un abordaje integral con distintos enfoques y niveles como se viene haciendo por parte de la SEMES:

- Un enfoque de sistema.

- Una visión operativa, de gestión clínica, a nivel de las unidades: servicios o secciones clínicas.

- Una perspectiva técnica que permita mediciones precisas para la adopción de medidas directas sobre los procesos de asistencia directa a los pacientes, o sea de indicadores de calidad.

\section{CONCEPTO DE ACREDITACIÓN}

La acreditación de centros y servicios sanitarios es, sin duda, el método de garantía de calidad más aceptado y constituye hoy en día una firme recomendación por parte de la administración sanitaria. Cada vez aparece más reglamentado este aspecto en la legislación española actual ${ }^{18-20}$. También podemos añadir que constituye una recomendación por parte de las sociedades científicas, y en particular, de la SEMES.

Podemos definir la acreditación como un proceso de evaluación externa al que se somete una organización sanitaria, basado en la revisión de unos criterios o estándares consensuados cuyo cumplimiento manifiesta que ese centro desarrolla sus funciones con parámetros de calidad, al menos, cercanos a la excelencia. Recordemos que, en definitiva, acreditar no es más que "hacer algo digno de crédito" ${ }^{21}$.

Para entender mejor el concepto, debemos hacer unas aclaraciones, al menos de aquellos conceptos que causan mayor confusión. No debemos confundir acreditación con:

- Autorización: acto y documento por el cual se permite la realización de una actividad. Ésta emana de un poder que ejerce el gobierno y, por lo 
tanto, se hace obligatoria y previa al inicio del ejercicio correspondiente.

- Inspección: significa realizar un examen o reconocimiento con una finalidad fiscalizadora, para comprobar el cumplimiento de una normativa que se exige para desarrollo de una actividad. También es obligatoria.

- Certificación: certificar es hacer constar por escrito una realidad de hecho por quien tenga fe pública o atribución para ello. Es por ello que la mayoría de las organizaciones acreditadoras extienden como colofón del proceso un documento certificando que se ha superado la evaluación llevada a cabo. En ocasiones se utiliza indistintamente acreditación o certificación.

$\mathrm{Al}$ menos existen dos son premisas aceptadas universalmente respecto a la acreditación:
1. Que sea voluntaria, lo que la distingue de la autorización y homologación.

2. Que sea llevada a cabo por una entidad ajena a la propia institución.

De la segunda premisa se derivan las exigencias que debe tener la entidad acreditadora: capacidad generadora de consenso y aceptación respecto a los criterios de calidad que se evalúan, prestigio suficiente para transmitir una distinción y suficiente independencia de modo que no tenga ningún tipo de interés cruzado con la entidad a acreditar.

Así pues, el fundamento de todos los sistemas de acreditación consiste en someter la organización que quiere ser acreditada a unas normas conocidas, por una organización independiente. Este modelo se repite en todos los sistemas y son característicos de cada uno las normas y el sistema de evaluación.

Tabla 1. Sistemas de acreditación de la calidad.

\begin{tabular}{ll}
\hline Globales para & - ISO (1947) \\
todos los sectores & - EFQM (1989) \\
económicos & - Premios Malcolm Baldrige (EE.UU 1988) \\
& - Premios Deming (Japón 1951) \\
& - Joint Commission on Accreditation of Healthcare Organizations. 1951 \\
& - Canadian Commission on Hospital Accreditation \\
Generales & - King's Found (Inglaterra. Auditoría desde 1980) \\
para Servicios & - Australian Council on Healthcare Standards \\
Sanitarios & - Agencia Calidad Sanitaria Andalucía (ACSA 2002) \\
& - INACEPS (Comunidad Valenciana 2002) \\
& - Otros \\
\hline & - JACIE. Joint Accreditation Committee-ISCT \& EBMT. 1998 Trasplante células madre \\
& hematopoyéticas. \\
Específicos de & - ONT. Sistema de Acreditación de. Organización nacional de Transplantes dirigido a \\
Sociedades & centros con unidades con capacidad de realización extracción y transplantes de órganos \\
Científicas e & y tejidos. 2000. \\
Instituciones & - SEMES. Sistema de Acreditación para Servicios de Urgencias, Emergencias y urgencias en \\
& Atención primaria. 2004. \\
& - SEN. Sistema de Acreditación de la Sociedad Española de Neurología. 2006. \\
& - AEEH. Sistema de Acreditación de la Asociación Española de las Enfermedades hepáticas. 2009. \\
\hline
\end{tabular}

\section{EVOLUCIÓN DE LOS SISTEMAS DE ACREDITACIÓN/CERTIFICACIÓN Y SUS DIFERENTES MODELOS}

Podemos considerar la publicación, por parte del Colegio Americano de Ciru- janos en 1917 de unos criterios mínimos (The Minimun Standars) en los EEUU para permitir el desarrollo de la cirugía en los hospitales, el germen de la acreditación. El éxito de esta iniciativa llevó posteriormente a la agrupación de los diferentes grupos 
médicos a crear una organización mayor con un claro fin acreditador: Joint Comission on Accreditation of Hospitals (JCAH). A partir de esta organización sanitaria, en constante evolución y ligada siempre a aspectos de calidad sanitaria, se crea en 1994 la Joint Comission International (JCI) (www. jointcommissioninternational.org), entidad en la que hoy encontramos localizada la actividad acreditadora a nivel internacional de esta organización y que constituye un pilar básico para la comprensión de los sistemas de acreditación en el mundo sanitario.

Otro pilar sobre el que asienta la comprensión de los sistemas de certificación/ acreditación es la creación en 1947 de las ya citadas normas ISO (www.iso.org).

Si bien de la JCI podemos destacar su clara vinculación sanitaria, que la hace cercana a los profesionales, de las normas ISO, con menor capacidad de adaptación al mundo sanitario, debemos indudablemente destacar su reconocimiento mundial fundamentado en una base extremadamente sólida de credibilidad, ya que todas las agencias que aplican las normas ISO deben a su vez estar acreditadas por otras agencias que están basadas en su propia nor- mativa. En España es ENAC (www.enac. es), auspiciada y tutelada por la Administración, la agencia designada para evaluar la competencia técnica de los organismos de evaluación que aplican esta normativa. Entre las entidades acreditadas por ENAC habría que destacar por su relevancia $\mathrm{AE}$ NOR (www.aenor.org como una de las de mayor prestigio y actividad acreditadora.

Hay que mencionar un tercer modelo para la comprensión de los sistemas de acreditación, un sistema especial de gestión y mejora de la calidad creado en Europa en 1989: la Fundación Europea para la Gestión de la Calidad, conocida internacionalmente por sus siglas del inglés EFQM. Este modelo se ha adaptado posteriormente a empresas públicas y ha llegado al ámbito sanitario con gran aceptación. Si bien por su origen industrial adolece del mismo problema que atribuimos a las normas ISO, y su inicio mucho más reciente no le permite adjudicarle la experiencia de las otras dos organizaciones, hay que destacar en este sistema, junto con el indudable acierto de la agrupación de sus llamados "agentes» y "resultados» fácilmente adaptables al mundo sanitario, tiene un punto fuerte en el aspecto de la potenciación de la autoevaluación.

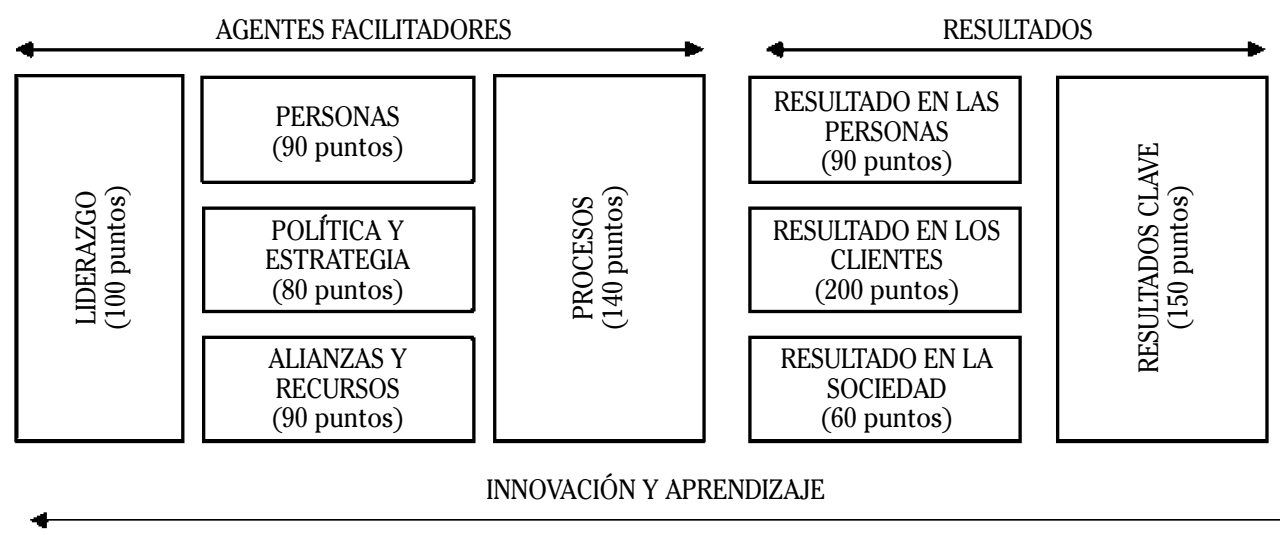

Figura 1. Criterios del Modelo EFQM de excelencia empresarial.

Es a partir del año 2000 cuando las sociedades científicas comienzan a incidir en el ámbito de la acreditación, retomando aquel ejemplo de los cirujanos en EEUU y reclamando ser papel fundamental en la determinación de los aspectos de calidad en sus respectivos campos profesionales específicos. Lógicamente debemos desta- 
car aquí el papel fundamental que para los servicios de urgencias y emergencias tiene la creación por parte de la SEMES de un sistema propio de acreditación de centros y servicios de urgencias que ya hoy tiene en su haber la evaluación de casi una decena de centros, con tres centros hospitalarios y un sistema de emergencias acreditados.

Existen otras agencias de acreditación arraigadas en otros países como en Inglaterra (King`s Found) o en Canadá o Australia (Canadian Comission y Australian Council), con grandes similitudes con el modelo americano de la Joint Comisión y que suscitan interés fundamentalmente en esos países.

En España, pese a que prácticamente todas las comunidades autónomas han desarrollado mecanismos para fomentar la acreditación de sus centros, fundamentalmente mediante la utilización de los sistemas citados, nos parecen destacables los que se citan a continuación.

La Agencia de Calidad Sanitaria de Andalucía es una fundación pública adscrita a la Consejería de Salud de la Junta de Andalucía. Ha desarrollado unos programas y manuales de acreditación (publicados en su página web: www.juntadeandalucia.es, agenciadecalidadsanitaria/programas_de_ acreditacion) y realiza a través de los equipos de evaluadores de la propia agencia una evaluación externa.

El sistema de acreditación en Cataluña está regulado por ley. Se realiza una evaluación de los estándares publicados previamente y divulgados también en su página web (www.gencat.net) y se realiza una evaluación externa a través de entidades ajenas a la administración pero que previamente han sido acreditadas por ésta. Los criterios incluyen algunos referidos a la asistencia urgente.

En la Comunidad Valenciana, la Generalitat Valenciana ha impulsado el desarrollo de una institución específica: el Instituto para la Acreditación y Evaluación de las Prácticas Sanitarias (INACEPS). La administración mantiene una participación a través de su consejo de dirección, aunque le imprime un carácter de independencia al instituto a través de la elaboración de sus estándares de forma muy participativa con las sociedades científicas. Se está desarrollando un manual específico para servicios de urgencias.

En Galicia se estableció en 2001 un sistema de acreditación de centros hospitalarios desde la propia administración sanitaria. Hace referencia a las unidades de urgencias hospitalarias señalando unos requisitos para el área de urgencias acordes con la complejidad de los servicios que ofrece el hospital.

En Extremadura existe un sistema de acreditación propio desde 2005, basado en el modelo EFQM, dependiente de la Consejería de Sanidad, de modo similar al de Andalucía.

\section{CALIDAD Y ACREDITACIÓN EN NUESTRO PAÍS}

En nuestro país, la SEMES ha sido pionera en el desarrollo tanto de estándares de calidad como de sistemas de acreditación para los diferentes eslabones que componen la cadena de la atención sanitaria urgente. En los últimos años han sido numerosos los libros editados por nuestra sociedad en una apuesta clara por la calidad, fruto del esfuerzo de profesionales de emergencias, conscientes de la relevancia del proyecto.

Sin embargo, desde la puesta en marcha del sistema de acreditación de SEMES pocos han sido los servicios que la han obtenido (anexo). El motivo es, sin duda, la gran carga asistencial que soportan que impide su adecuada organización y la necesaria dedicación a otras tareas indispensables para el correcto funcionamiento de cualquier servicio.

Las plantillas son diseñadas en base a la actividad asistencial y es habitual que ya desde su inicio sean insuficientes para la misma. El creciente aumento de atenciones crea un círculo vicioso en el que más demanda (en la mayoría de los casos no urgente) genera algo más de recurso tras producir más caos y desmotivación entre 
el personal, al verse obligado a desarrollar una actividad laboral que en la mayoría de los casos no se corresponde con sus expectativas.

\section{FUTURO}

Sin duda el futuro es prometedor, aunque en determinados momentos nos cueste verlo. Dependerá en gran medida de la consecución de diferentes logros entre los cuales se encuentran:

1. Acreditación de los centros para hacer dignos de crédito nuestros servicios. Nos permite conocer la realidad de cada servicio y el camino que debe seguir si queremos tener la base para prestar una atención de calidad. Debería ser una obligación para la administración, y como tal exigírselo, el conocer el estado en el que están sus servicios de urgencias y, hoy por hoy, la mejor herramienta sin duda es ésta.

2. Reconocimiento de la especialidad de Medicina de Urgencias y Emergencias. Es una garantía tanto para la población como para la administración, además de ser la solución de continuidad de nuestros servicios. Sin duda contribuirá a la homogenización de la atención dejando definido el papel de estos servicios en la sociedad.

3. Ordenamiento de la atención urgente. Es indispensable frenar el crecimiento asistencial de esta actividad y poner orden en nuestros servicios. La administración junto con los propios trabajadores debe comprometerse en regular la atención urgente. Se debe evitar que lleguen a nuestros servicios pacientes cuyos problemas se pueden y deben tratar en otros puntos de la red asistencial. El resultado final sería más calidad en nuestras asistencia, más motivación en nuestros profesionales y, lo más importante, mejor atención para los ciudadanos.
4. Gestión por procesos. Es necesario definir los procesos y subprocesos para medirlos y compararlos y así poder mejorarlos periódicamente. Es una herramienta básica de calidad además de indispensable para lograr una mejora continua.

5. Consolidación de las unidades de gestión clínica. Los servicios a lo largo del tiempo han mostrado su madurez. Es necesario dar un paso en su autonomía para delegar en ellos la responsabilidad de gestionarse, lo cual sin duda mejorará el uso de los recursos y contribuirá a generar confianza y motivación en los profesionales, indispensable en servicios de estas características.

Para llevar a cabo estos objetivos no sólo es imprescindible el concurso de los servicios de urgencias, sino también de la administración y de las propias direcciones de los centros. Los servicios de urgencias entonces dejarán de ser un cajón de sastre donde cabe todo para así poder dedicarse a lo que le es propio y constitutivo: la atención urgente.

\section{BIBLIOGRAFÍA}

1. Haynes AB, Weiser TG, BerRy WR, Lipsitz SR, BREIZAT AH, DelLingER EP et al. A surgical safety checklist to reduce morbidity and mortality in a global population. N Engl J Med 2009; 360: 491-499.

2. Ministerio de Sanidad y Consumo. Estudio Nacional sobre los Efectos Adversos ligados a la Hospitalización. ENEAS 2005 Informe 2006.

3. SAlgado E. Gestión de riesgos. Rev Calidad Asistencial 2005; 20: 49-50.

4. Tejedor M, Martín JM. Sistema de calidad en un servicio de emergencias sanitarias. Rev Calidad Asistencial 1999; 14: 671-679.

5. Estándares de acreditación para servicios de urgencias de hospitales. Sociedad Española de Medicina de Urgencias y Emergencias. $2^{\mathrm{a}}$ Edición. 2008. ISBN: 978-84-87450-17-4.

6. Estándares de acreditación para servicios de emergencias. Sociedad Española de Medicina de Urgencias y Emergencias. Junio, 2006. ISBN: 84-87450-96-2. 
7. Estándares de Acreditación para empresas proveedoras de Servicios de Emergencias. Sociedad Española de Medicina de Urgencias y Emergencias. Edición 2009. ISBN: 97884-87450-34-1.

8. Estándares de acreditación para Servicios de Urgencias de Atención Primaria. Sociedad Española de Medicina de Urgencias y Emergencias. Edición 2009. ISBN: 978-84-87450-31-0.

9. López-Andújar L, Aljama Alcántara M, PerezMontaud Merino I, Cartelle Pérez T, Casado Martínez JL, Tomás Vecina S. Acreditación de Servicios de Urgencias y Emergencias. Todo Hospital 2003; 198: 456-61.

10. Clemente Ricote G, Pérez lázaro JJ, Tejedor FernÁNDEZ M, ArRoyo Pérez V y Grupo de Estudio de Calidad en Hepatología de la Asociación Española para el Estudio del Hígado (AEEH). Sistema Español de Acreditación de Unidades de Hepatología. Una propuesta de la Asociación Española para el Estudio del Hígado. Gastroenterol Hepatol 2007; 30: 612-617.

11. Clemente G, Pérez-Lázaro JJ, Tejedor M, Planas R, De la Mata M, Córdoba J et al. Acreditación de procesos en hepatología. Gastroenterol Hepatol 2008; 31: 427-432.

12. Clemente Ricote G, Pérez-Lázaro JJ, Tejedor M, Planas R, de la Mata M, Córdoba J et al. Sistema Español de Acreditación de Competencias Profesionales en Hepatología. Una propuesta de la Asociación Española para el Estudio del Hígado. Gastroenterol Hepatol 2008; 31: 530-535.
13. Tejedor Fernández M, Pérez Lázaro JJ, García Alegría J. Gestión clínica: Aplicación práctica en una Unidad Hospitalaria (II). Rev Calidad Asistencial 2003; 18: 125-131.

14. Pérez lázaro JJ, García Alegría J, Tejedor FerNÁNDEZ M. Gestión clínica: conceptos y metodología de aplicación. Rev Calidad Asistencial 2002; 17: 305-311.

15. Tejedor Fernández M, Burillo Putze G, Roqueta Egea F, Jiménez Murillo L, Leciñena Esteban MA, Carretero Alcántara L. Gestión clínica en un Servicio de Urgencias. Todo Hospital 2003; 198: 462-470.

16. Díaz Fernández JL, Arce Mateos FP, Tejedor FerNÁNDEZ M. Gestión de la actividad médica. En: Temes, JL y Mengíbar M, editores. Gestión Hospitalaria. Cuarta Edición. EDIT. McGrawHill. interamaericana. 2007. Capítulo 7. Págs. 81-96. ISBN: 978-84-481-5573-5.

17. Manual de Indicadores para los Servicios de Urgencias de Hospitales. Sociedad Española de Medicina de Urgencias y Emergencias. Edición 2009. ISBN: 978-84-87450-30-3.

18. Ley 14/1986 General de Sanidad (BOE 101/1986 de 29-04-1986). Capítulo II

19. Ley 16/2003 de Cohesión y Calidad del Sistema Nacional de Salud. Apartado VII. Artículo 28 , artículo 60 y artículo 62 .

20. Plan de Calidad del Sistema Nacional de Salud. Estrategia 7.

21. Diccionario de la Lengua Española. Real Academia Española de la Lengua. 2001. Tomo I 